\title{
Probing the QCD phase diagram with measurements of strange hadron elliptic flow in STAR
}

\author{
Md. Nasim (for the STAR collaboration)* \\ Department of Physics \& Astronomy \\ University of California, Los Angeles \\ CA-90095, USA \\ E-mail: nasimercf.rhic.bnl.gov
}

\begin{abstract}
We present the measurements of strange hadron elliptic flow at mid-rapidity in $\mathrm{Au}+\mathrm{Au}$ collisions at $\sqrt{s_{N N}}=7.7-200 \mathrm{GeV}$ using the STAR detector in the years 2010 and 2011. The transverse momentum and collision centrality dependence of elliptic flow is presented. At the intermediate transverse momentum $\Omega$ baryon and $\phi$-meson show baryon-meson separation effect similar to proton and pion for minimum-bias $\mathrm{Au}+\mathrm{Au}$ collision at $\sqrt{s_{N N}}=200 \mathrm{GeV}$. This indicates formation of collective flow at the early partonic phase. The separation between baryons and mesons at intermediate transverse momentum decreases with decrease in beam energy and almost disappears at $\sqrt{s_{N N}} \leq 11.5 \mathrm{GeV}$, indicating hadronic interaction being dominant at the lower beam energy. We observe difference in elliptic flow between particle and anti-particle and this increases with decrease in beam energy. Differences are larger for baryons than mesons. The relative difference between particle and anti-particle elliptic flow is larger in central collisions than in peripheral ones.
\end{abstract}

7th International Conference on Physics and Astrophysics of Quark Gluon Plasma,

1-5 February, 2015

Kolkata, India

${ }^{*}$ Speaker. 


\section{Introduction}

According to quantum chromodynamics (QCD) at very high temperature and/or at high density, a de-confined phase of quarks and gluons is expected to be present, while at low temperature and low density the quarks and gluons are known to be confined inside hadrons. The aim of the STAR experiment at Relativistic Heavy Ion Collider (RHIC) is to study the QCD matter by colliding nuclei at ultra-relativistic speeds [1]. Using the information carried by freely streaming final-state particles as probes, we try to understand the properties of the medium created in these collisions. Strange particle elliptic flow $\left(v_{2}\right)$ is one of the observables that is expected to deliver detailed information on the reaction dynamics of relativistic nucleus-nucleus collisions [2, 3, 4]. In high energy heavy-ion collisions, particles are produced with an azimuthally anisotropic momentum distribution. Elliptic flow is a measure of azimuthal angle $(\varphi)$ anisotropy of the produced particles with respect to the reaction plane angle $(\psi)$. The elliptic flow is believed to arise due to the pressure gradients developed in the overlap region of the two nuclei colliding at nonzero impact parameter. The $v_{2}$ is an early time phenomenon and expected to be sensitive to the equation of state of the system formed in the collision [5]. Thus $v_{2}$ can be used as a probe for early system although its magnitude may change due to later-stage hadronic interactions. The interaction cross-sections of the multi-strange hadrons $(\Xi, \Omega)$ and $\phi$-meson with non-strange hadrons are expected to have a small value and therefore its production should be less affected by the later stage hadronic interactions in the evolution of the system formed in heavy-ion collisions $[6,7,8]$. Moreover they seem to freeze-out early than non-strange hadrons [9]. Therefore, multi-strange hadrons can be considered as a clean probe to study the QCD matter.

\section{Data sets and methods}

The results presented here are based on data collected at $\sqrt{s_{N N}}=7.7,11.5,19.6,27,39,62.4$ and $200 \mathrm{GeV}$ in $\mathrm{Au}+\mathrm{Au}$ collisions by the STAR detector for a minimum bias trigger in the years of 2010 and 2011. The Time Projection Chamber (TPC) and Time of Flight (TOF) detectors with full $2 \pi$ coverage are used for particle identification in the central pseudo-rapidity $(\eta)$ region $(|\eta|<$ 1.0). We reconstruct short-lived $K_{S}^{0}, \Lambda(\bar{\Lambda}), \Xi^{-}\left(\bar{\Xi}^{+}\right), \Omega^{-}\left(\bar{\Omega}^{+}\right)$and $\phi$ through the following decay channels : $K_{S}^{0} \rightarrow \pi^{+}+\pi^{-}, \Lambda \rightarrow p+\pi^{-}\left(\bar{\Lambda} \rightarrow \bar{p}+\pi^{+}\right), \Xi^{-} \rightarrow \Lambda+\pi^{-}\left(\bar{\Xi}^{+} \rightarrow \bar{\Lambda}+\pi^{+}\right), \Omega^{-} \rightarrow \Lambda$ $+K^{-}\left(\bar{\Omega}^{+} \rightarrow \bar{\Lambda}+K^{+}\right)$and $\phi \rightarrow K^{+}+K^{-}$. Mixed event technique has been used for combinatorial background estimation [10] as shown in Fig 1. The $\eta$-sub event plane method [11] using TPC tracks has been applied to measure the elliptic flow. In this method, one defines the event flow vector for each particle based on particles measured in the opposite hemisphere in pseudo-rapidity $(\eta)$

$$
v_{2}\left(\eta_{ \pm}\right)=\frac{<\cos \left[2\left(\phi_{\eta_{ \pm}}-\psi_{2, \eta_{\mp}}\right)\right]>}{\sqrt{<\cos \left[2\left(\psi_{2, \eta_{+}}-\psi_{2, \eta_{-}}\right)\right]>}} .
$$

Here $\psi_{2, \eta_{+}}\left(\psi_{2, \eta_{-}}\right)$is the second harmonic event plane angle defined for particles with positive(negative) pseudo-rapidity. An $\eta$ gap of $\Delta \eta=0.1$ between positive and negative pseudo-rapidity sub-events has been introduced to suppress non-flow effects. 

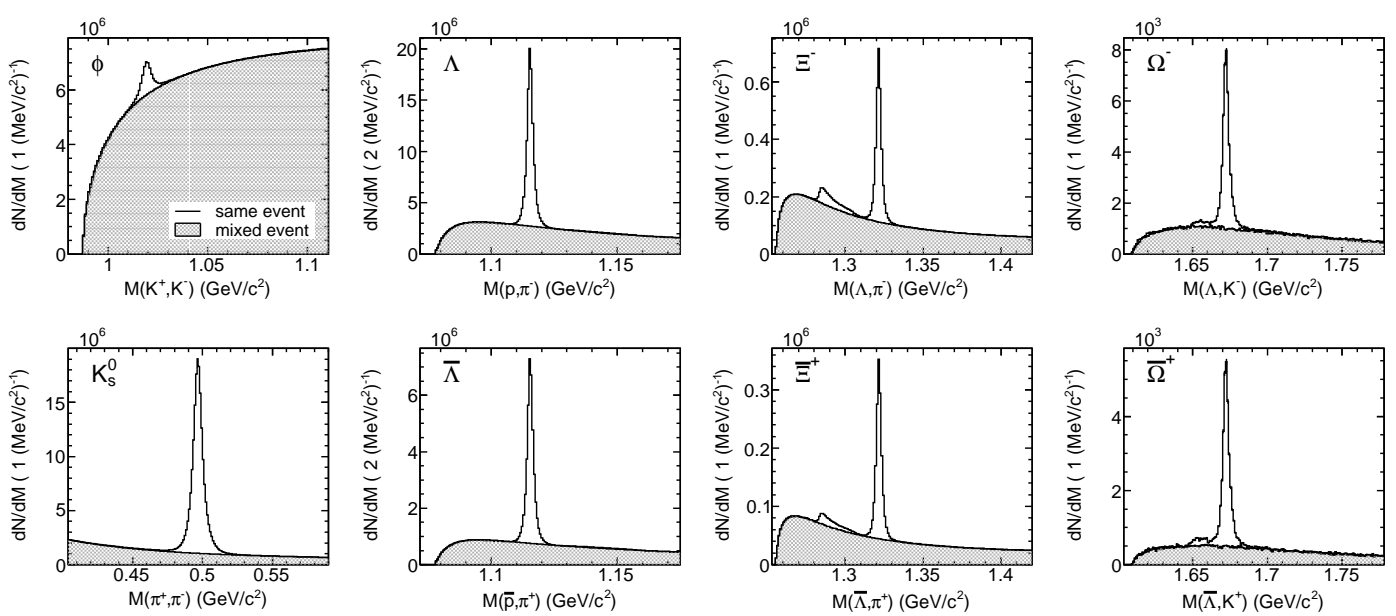

Figure 1: (Color online) Examples of the invariant mass distributions at $\sqrt{s_{N N}}=62.4 \mathrm{GeV}$ for $\phi, K_{S}^{0}, \Lambda(\bar{\Lambda})$, $\Xi^{-}\left(\bar{\Xi}^{+}\right)$and $\Omega^{-}\left(\bar{\Omega}^{+}\right)$. The combinatorial background is described by the mixed-event technique.

\section{Results}

\subsection{Elliptic Flow at Top RHIC Energy}

The number of constituent quarks (NCQ) scaling in $v_{2}$ for different identified hadrons has been considered as a good probe for studying the strongly interacting partonic matter. The observed NCQ scaling of identified hadrons in experimental data [12] indicates the importance of parton recombination in forming hadrons in the intermediate $p_{T}$ range $\left(2.0 \mathrm{GeV} / \mathrm{c}<p_{T}<4.0\right.$ $\mathrm{GeV} / \mathrm{c})[13,14,15]$. Such scaling may indicate that collective elliptic flow is developed during the partonic phase. The large statistics data sets collected by STAR detectors allow us to measure elliptic flow of multi-strange hadrons, specifically that of the $\Omega$ baryon which is made of pure strange $(s)$ or anti-strange $(\bar{s})$ constituent quarks and of the $\phi$ meson, consisting of one $s$ and one $\bar{s}$ constituent quark. Fig. 2 shows the $v_{2}$ as a function of $p_{T}$ for $\pi, p, \phi$ and $\Omega$ for $0-80 \%$ centrality

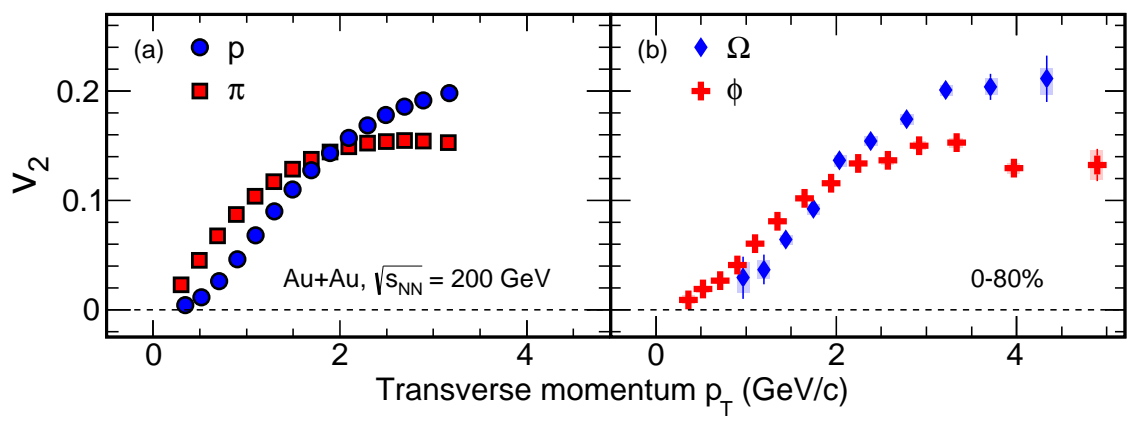

Figure 2: (Color online) The $v_{2}$ as function of $p_{T}$ for $\pi, p$ (panel a) and $\phi, \Omega$ (panel b) from minimum bias $\mathrm{Au}+\mathrm{Au}$ collisions at $\sqrt{s_{N N}}=200 \mathrm{GeV}$ for $0-80 \%$ centrality [16]. The systematic uncertainties are shown by the shaded boxes while vertical lines represent the statistical uncertainties.

in $\mathrm{Au}+\mathrm{Au}$ collisions at $\sqrt{s_{N N}}=200 \mathrm{GeV}$ [16]. Fig. 2 (a) shows a comparison between $v_{2}$ of $\pi$ 
and $p$, consisting of up $(u)$ and down $(d)$ light quarks, and Fig. 2 (b) shows a comparison of $v_{2}$ of $\phi$ and $\Omega$ containing heavier $s$ quarks. The $v_{2}$ of $\phi$ and $\Omega$ are mass ordered at low $p_{T}$ and a baryonmeson separation is observed at intermediate $p_{T}$. It is clear from Fig. 2 that the $v_{2}\left(p_{T}\right)$ of hadrons consisting only of strange quarks ( $\phi$ and $\Omega$ ) is similar to that of $\pi$ and $p$. However, unlike $\pi$ and $p$, the $\phi$ and $\Omega$ do not participate strongly in the hadronic interactions, which suggests that the major part of collectivity is developed during the partonic phase in $\mathrm{Au}+\mathrm{Au}$ collisions at $\sqrt{s_{N N}}=200 \mathrm{GeV}$.

Fig. 3 shows the $v_{2}$ scaled by number of constituent quarks $\left(n_{q}\right)$ as a function of $p_{T} / n_{q}$ and $\left(m_{T}-m_{0}\right) / n_{q}$ for identified hadrons from $\mathrm{Au}+\mathrm{Au}$ collisions at $\sqrt{s_{N N}}=200 \mathrm{GeV}$ for $0-30 \%$ and $30-80 \%$ centrality [16], where $m_{T}$ and $m_{0}$ are the transverse mass and rest mass of hadron, respectively. To quantify the deviation from NCQ scaling, we fit the $K_{S}^{0} v_{2}$ with a third-order polynomial function. We then take the ratio of $v_{2}$ for the other measured hadrons to the $K_{S}^{0}$ fit. The ratios are shown in the lower panels of Fig. 3. For both $0-30 \%$ and 30-80\% centralities, the scaling holds approximately within $10 \%$, excluding pions. The deviation of pions could be due the effect of resonance decay and non-flow correlations [17].

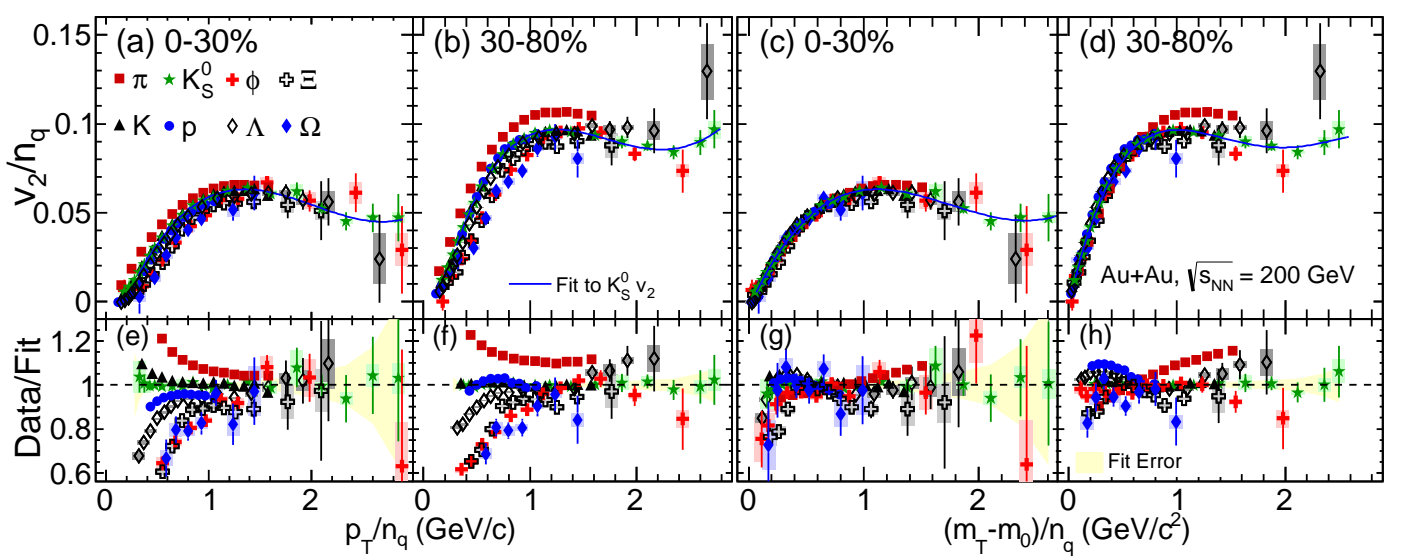

Figure 3: (Color online) The $v_{2}$ scaled by number of constituent quarks $\left(n_{q}\right)$ as a function of $p_{T} / n_{q}$ and $\left(m_{T}-m_{0}\right) / n_{q}$ for identified hadrons from $\mathrm{Au}+\mathrm{Au}$ collisions at $\sqrt{s_{N N}}=200 \mathrm{GeV}$ [16]. Ratios with respect to a fit to the $K_{S}^{0} v_{2}$ are shown in the corresponding lower panels. Vertical lines are statistical uncertainties and shaded boxes are systematic uncertainties.

\subsection{Elliptic Flow at Beam Energy Scan}

\subsubsection{Elliptic flow as a function of transverse mass}

Fig. 4 shows $v_{2}$ as function of $\left(m_{T}-m_{0}\right)$ at $\sqrt{s_{N N}}=7.7-62.4 \mathrm{GeV}[18,19]$. There is a clear splitting between baryons and mesons for larger $\left(m_{T}-m_{0}\right)$ values at $\sqrt{s_{N N}}=62.4 \mathrm{GeV}$. As we go down in energy, the splitting becomes narrower and at $11.5 \mathrm{GeV}$, the difference between the baryons and mesons is no longer observed. Also, we observed that $v_{2}$ of $\phi$ mesons falls off the trend from the other hadrons at $\sqrt{s_{N N}} \leq 11.5 \mathrm{GeV}$. This could be related to the lower hadronic cross sections of particles containing multiple strange quarks $[6,7,8]$. These observations may indicate that hadronic interactions become more important than partonic ones for the systems formed at collision energies $\sqrt{s_{N N}} \leq 11.5 \mathrm{GeV}$. 


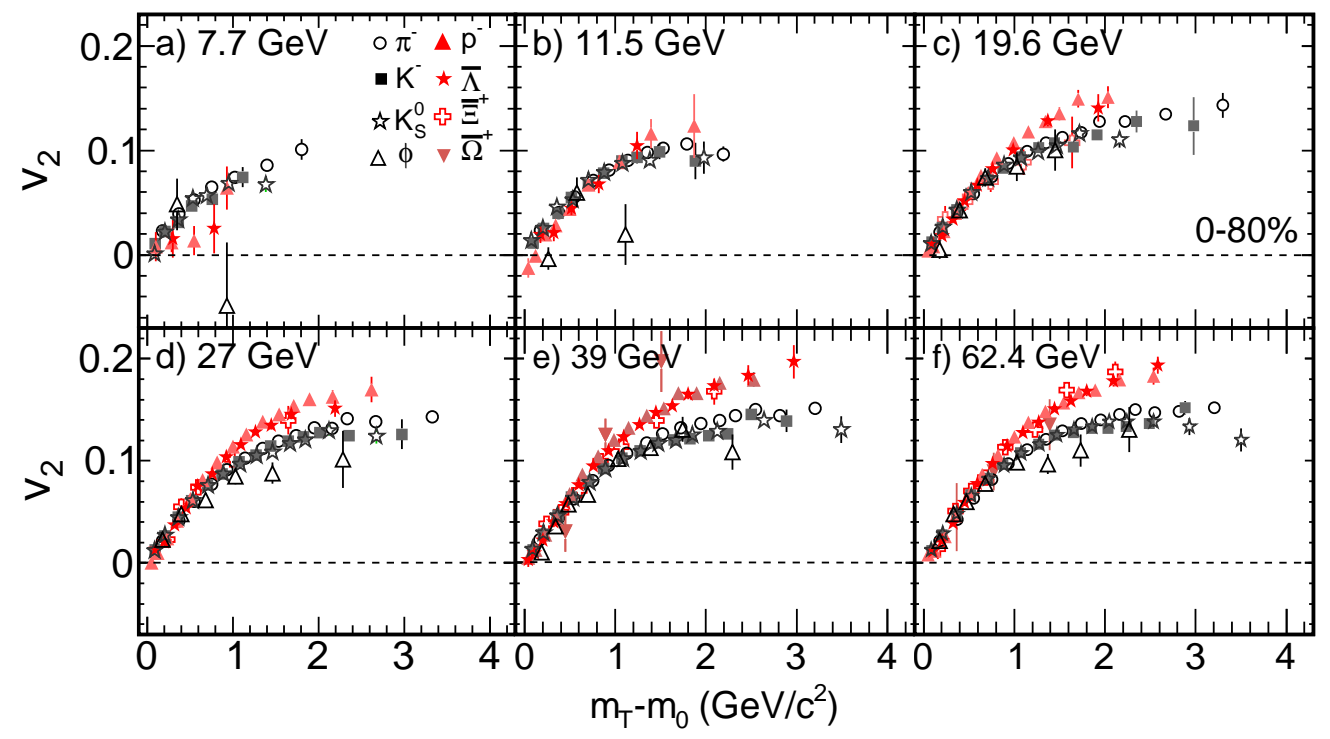

Figure 4: (Color online) The elliptic flow $\left(v_{2}\right)$ as a function of $\left(m_{T}-m_{0}\right)$ for selected particles in the $\mathrm{Au}+\mathrm{Au}$ collision at various beam energies for $0-80 \%$ centrality $[18,19]$. Error bars are only statistical uncertainties.

\subsubsection{Energy and centrality dependence of the particle and antiparticle $v_{2}$ difference}

The energy dependence of the $v_{2}$ difference between particles and antiparticles for different centralities are shown in Fig. 5. Top panel shows the differences for $\pi, K, p, \Lambda$ and $\Xi$ at $10-40 \%$ collisions centrality. We can see that the difference for all baryons are same at $10-40 \%$ centrality for all $\sqrt{s_{N N}}$, which is consistent with the observation at 0-80\% $[18,19]$. Middle and lower panel of Fig. 5 show the differences between protons and anti-protons for $0-10 \%, 10-40 \%$ and $40-80 \%$ centralities. The y-axis of the lower panel is scaled by the proton $v_{2}$ at $p_{T}=1.5 \mathrm{GeV} / c$ (labeled as $v_{2}^{\text {norm }}$ ) to show the relative difference. We can see, the relative difference in $v_{2}$ between protons and anti-protons increases from peripheral (40-80\%) to central (0-10\%) collisions. This observation support the model prediction [20], which includes baryon stopping as a mechanism to explain the data.

\section{Summary}

Energy and centrality dependence of strange hadron $v_{2}$ at mid-rapidity are presented. The $p_{T}$ dependence of $\phi$ and $\Omega v_{2}$ is similar to $\pi$ and $p v_{2}$ at top RHIC energy, which indicates that the major parts of collectivity were developed at the initial partonic phase at $\sqrt{s_{N N}}=200 \mathrm{GeV}$. To investigate partonic collectivity for different system size, NCQ scaling has been presented for two different collision centralities, $0-30 \%$ and $30-80 \%$. It is observed that the NCQ scaling holds within the statistical uncertainty for both $0-30 \%$ and 30-80\% centralities. Splitting between baryon and meson at intermediate $p_{T}$, which formed the basis of NCQ scaling observation at top RHIC energy, was not observed at the lower energies indicating formation of a matter mostly governed 


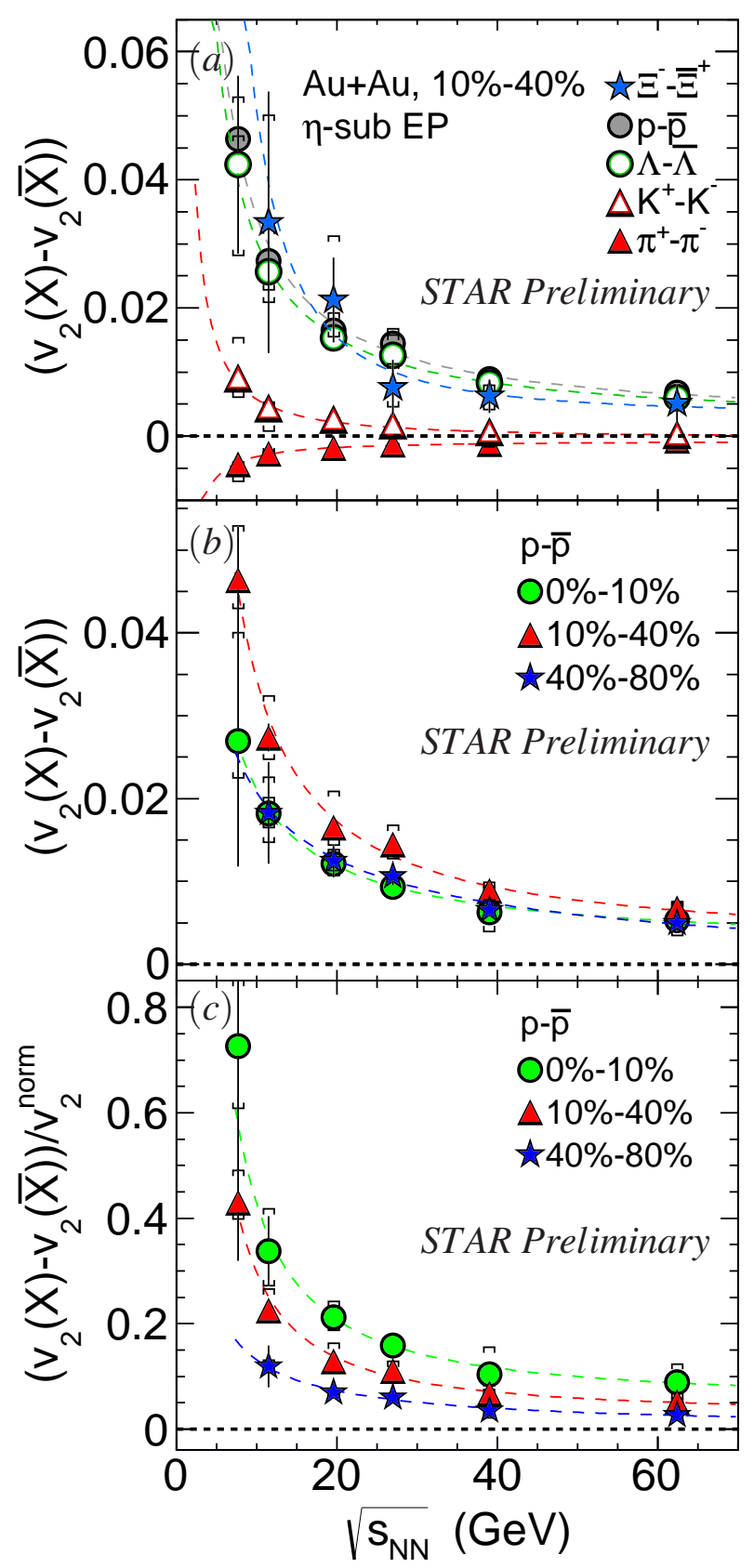

Figure 5: (Color online) The difference in $v_{2}$ values between a particle $(X)$ and its corresponding antiparticle $(\bar{X})$ as a function of $\sqrt{s_{N N}}$. The dashed lines in plot are fits using function $f_{\Delta v_{2}}\left(\sqrt{s_{N N}}\right)=a \times s_{N N}^{-b / 2}$. Here $v_{2}^{\text {norm }}$ is equal to proton $v_{2}$ at $p_{T}=1.5 \mathrm{GeV} / c$.

by hadronic interaction. We observed beam-energy dependent difference in $v_{2}$ between particle and corresponding anti-particle. Differences are larger for baryons than mesons. The difference 
increases with decreasing beam energy. Relative difference between particle and anti-particle $v_{2}$ is larger in central collisions than in peripheral ones.

\section{Acknowledgements}

Financial support from DOE project is gratefully acknowledged.

\section{References}

[1] J. Adams et al. ( STAR Collaboration) Nuclear Physics A 757, 102 (2005).

[2] B. I. Abelev et al. (STAR Collaboration) Phys. Rev. C 77, 054901 (2008).

[3] B. I. Abelev et al. (STAR Collaboration) Phys. Rev. C 81, 044902 (2010).

[4] B. B. Abelev et al. (ALICE Collaboration) JHEP 1506, 190 (2015).

[5] P. F. Kolb et al. Nucl. Phys. A 715, 653c (2003).

[6] A. Sibirtsev et al., Eur. Phys. J. A 29, 209 (2006).

[7] B. Mohanty and N. Xu , J. Phys. G 36, 064022 (2009).

[8] Md. Nasim, B. Mohanty and N. Xu, Phys. Rev. C 87, 014903 (2013).

[9] A. Shor, Phys. Rev. Lett. 54, 1122 (1985).

[10] B. I. Abelev et al. (STAR Collaboration) Phys. Lett. B 673, 183 (2009).

[11] A. M. Poskanzer and S. A. Voloshin, Phys. Rev. C 58, 1671 (1998).

[12] B. I. Abelev et al. (STAR Collaboration) Phys. Rev. Lett. 99, 112301 (2007).

[13] D. Molnar and S. A. Voloshin, Phys. Rev. Lett. 91, 092301 (2003).

[14] R. J. Fries et al. Phys. Rev. Lett. 90, 202303 (2003).

[15] L. X. Han et al. Phys. Rev. C 84, 064907 (2011).

[16] L. Adamczyk et al. (STAR Collaboration) arXiv: 1507.05247[nucl-ex].

[17] X. Dong et al. Phys. Lett. B 597, 328 (2004).

[18] L. Adamczyk et al. (STAR Collaboration) Phys. Rev. C 88, 014902 (2013).

[19] L. Adamczyk et al. (STAR Collaboration) Phys. Rev. Lett. 110, 142301 (2013).

[20] J. Steinheimer, V. Koch and M. Bleicher, Phys. Rev. C 86, 044903 (2012). 